\title{
On the Role of Modularity in Evolutionary Dynamic Optimisation
}

\author{
Philipp Rohlfshagen and Xin Yao, Member, IEEE
}

\begin{abstract}
The field of evolutionary dynamic optimisation is concerned with the application of evolutionary algorithms to dynamic optimisation problems. In recent years, numerous new algorithms have been proposed to track the problem's potentially moving global optimum as closely as possible. A large proportion of these techniques attempts to exploit possible similarities between successive problem instances, primarily using previously found solutions as starting points for future instances: If the previous global optimum is in close proximity to the new global optimum (in the genotype space), such transfer of knowledge should allow the algorithm to locate the new global optimum in less time than a random restart may require. However, it is clear that distance alone may be insufficient to guarantee such computational savings. In this paper, we propose a simple framework that may be used to create bi-modular problems with a variable degree of epistasis. We subsequently study how the dependencies between the two modules may affect the difficulty (number of function evaluation required) of relocating the new global optimum. We find that, given a simple (1+1) EA, even a modest degree of linkage between the problem's otherwise independent modules may have a significant impact on these attributes.
\end{abstract}

\section{INTRODUCTION}

The field of evolutionary dynamic optimisation [4], [5], [17], [13], [8] is concerned with the application of evolutionary algorithms (EAs) to dynamic optimisation problems (DOPs). The specifications of DOPs change over time, necessitating the adaptation of existing solutions to maintain feasibility and satisfactory quality. Such scenarios are frequently encountered in industrial settings where uncertainties are commonplace and hence considerable effort has recently been devoted to solving DOPs. In traditional optimisation, given some (objective) function $f: X \rightarrow \mathbb{R}$, the performance of an algorithm is usually judged by the time it takes the algorithm to locate the function's global optimum (or some solution of satisfactory quality). If we assume that we are maximising, the global optimum is defined as the point $x^{\star}$ such that $f\left(x^{\star}\right) \geq f(x), \forall x \in X$. In the case of dynamic optimisation, one is given a function $f: X \times \mathbb{N} \rightarrow \mathbb{R}$, the output of which depends on time $t \in \mathbb{N}$. In this case, the algorithm is usually required to track the time-variant global optimum as closely as possible as time goes by: If the previously located global optimum is lost due to a change

Philipp Rohlfshagen is with the Centre of Excellence for Research in Computational Intelligence and Applications (CERCIA), School of Computer Science, University of Birmingham, Birmingham B15 2TT, United Kingdom (phone: +44 121414 5142; email: p.rohlfshagen@cs.bham.ac.uk).

Xin Yao is with the Centre of Excellence for Research in Computational Intelligence and Applications (CERCIA), School of Computer Science, University of Birmingham, Birmingham B15 2TT, United Kingdom (phone: +44 121414 3747; email: x.yao@cs.bham.ac.uk). in the problem's specifications, the algorithm is required to relocate the global optimum as quickly as possible ${ }^{1}$.

In order to improve the algorithm's performance, a large proportion of newly developed evolutionary techniques attempt to exploit possible similarities between successive problem instances. In particular, one of the fundamental motivations in the field is the transfer of knowledge from one problem instance in time to the next [5]. In the traditional black-box scenario, no prior knowledge exists regarding the problem instances to be optimised and the search is usually initiated from a randomly chosen search point. In the dynamic case, one hopes that the similarities between some of the problem instances encountered are sufficient to usefully employ the knowledge gained about one instance (by sampling the objective function) to another. This knowledge may be utilised in different ways (e.g., adjust the algorithm's paramters), but most efforts so far have focused on the distances between successive global optima, using previously found solutions as starting points for future instances. This is largely motivated by the generally accepted idea that sufficiently small changes should allow the algorithm to successfully restrict the search to the neighbourhood of the previous global optimum [5].

In this paper we are interested in the problem properties that are responsible, at least in some cases, for the difficulty associated with locating the new global optimum starting from the old. This issue is closely related to the effective distance of a point: Although for large classes of optimisation problems, a point's distance to the global optimum is usually correlated to the time taken to locate the optimum starting from that point, other (local) properties of the fitness landscape, such as deceptiveness, may significantly influence the behaviour of the algorithm. Distance alone may thus be insufficient to warrant computational savings and here we analyse under what circumstances such cases may arise. More specifically, we look at how the presence of modularity may affect such properties and propose a simple framework to generate modular versions of pseudo-Boolean functions with a tuneable degree of overlap (dependency) between the modules. We subsequently use this framework to analyse the behaviour of the $(1+1)$ EA on the NK fitness landscape (NK).

The remainder of this paper is structured as follows: First we discuss the potential impact of a point's distance to the global optimum on the time it may take an algorithm to reach the optimum starting from that point (section II). We then

\footnotetext{
${ }^{1}$ It should be noted that this is only one possible scenario. In other cases, practitioners may be interested in different behaviours such as the algorithm's degree of robustness.
} 
outline the concept of modularity in section III where we also motivate our work from a biological perspective. Section IV introduces a simple dynamic framework suitable for analysis and the remainder of the paper presents an empirical study that highlights the performance of the $(1+1)$ EA on the modular NK. Finally, the paper in concluded in section VII where we also discuss future work.

\section{Distance to the Optimum}

In classical black-box optimisation, the algorithm has no knowledge about the problem instances it encounters and subsequently, the search is initiated from a uniformly random search point. In the case of pseudo-Boolean functions, this initial point is, in expectation, a Hamming distance of $n / 2$ away from the global optimum and, with overwhelming probability, no less than $1 / 3 n$ bits away from the global optimum (see [16]). The distance to the global optimum plays a crucial role in the time taken by an algorithm to locate the optimum due to the implicit design of most EAs: In order to locate the global optimum, the algorithm needs to traverse the search space and the majority of EAs do so by generating samples in the neighbourhood of already accepted search points (i.e., those in the population). New search points are accepted if their f-values are no worse than the f-values found so far and it follows that this ubiquitous design (see [7]) makes the implicit assumption that higher f-values will lead to the global optimum.

Clearly, whenever these assumptions break down, the performance of the algorithm is degraded. This has been exploited to formulate measures of problem difficulty based on the relationship between a point's fitness and its distance to the optimum. A well-known example is the fitness-distance correlation (FDC) which measures the correlation between a point's f-value and its distance to the global optimum [9]. However, numerous counter-examples have been produced where the FDC fails to reliably indicate problem difficulty (e.g., short path problems). A somewhat different approach is taken by Borenstein and Poli [2] who highlight the importance of a point's effective distance (as opposed to actual distance) to the global optimum. The effective distance of a point is defined as the probability that, given the algorithm samples this point, the global optimum will be found within some time bound. Borenstein and Poli argue that the difficulty of a problem is then equivalent to the number of times the assumptions made by the algorithm (that fitness is an indicator of effective distance) are incorrect.

On average, we would expect the assumptions made by the algorithm to be true given the class of problems the algorithm is designed for and applied to. Indeed, it has been shown that over all functions closed under permutation (i.e., if the classes of functions the algorithm is applied to is not restricted in any way), any two algorithms will perform equally, precisely because exploitable structures that conform to the assumptions made by the algorithm are removed from this set (No Free Lunch; see [18], [7]). From a practical point of view, however, such a set of functions is not only unrealistic for its size but also because one is generally not interested in the set of all functions. Instead, practitioners concentrate on subsets of functions that are of interest such as knapsack or travelling salesman problems; here it might be possible to design superior algorithms. In dynamic optimisation, a similar trend is evident: Most practitioner make the assumption that the global optimum will, on average, move relatively little and that it may be beneficial to restrict the search to the vicinity of the previously found global optimum. In other words, given the algorithm has already sampled a previous problem instance by the time a change is encountered, "a natural attempt to speed up optimisation after a change would be to somehow use knowledge about the previous search space to advance the search after a change." [8, p 311].

The majority of work in evolutionary dynamic optimisation is motivated by this concept and most techniques attempt to utilise the position of the global optimum at time $T$ as an initial searhch point to locate the global optimum at time $T+1$. Let us assume the dynamics of the problem shift the global optimum to a new position such that $d_{H}\left(x_{T}^{\star}, x_{T+1}^{\star}\right)=$ $\rho n$ where $\rho \in(0,1]$ represents the magnitude of change. As we assume changes to be small, we assume $\rho \leq \frac{1}{3} n$ and in order to relocate the global optimum, the algorithm needs to invert all $\rho n$ bits that are incorrect. For the sake of simplicity, we assume all incorrect bits to appear in the prefix of the solution and all correct bits in the suffix. The global optimum at time $T$ may then be described as $x_{T}^{\star}=a b$ and the algorithm needs to find $x_{T+1}^{\star}=\bar{a} b$ where $\bar{a}$ is the inverse of $a$. Of course, the algorithm does not know which bits need to be inverted and since we assume that $|a|<\frac{1}{2}|b|$, the correct bits have a much higher chance to be inverted by the mutation operator. It is crucial to note that the algorithm is sampling the point $x_{T}^{\star}$ because of the f-values obtained during period $T$. Following a change of the problem, the point may thus not be a local optimum or a point that would otherwise be accepted by the algorithm if the search had started from scratch at the beginning of period $T+1$.

In this paper we look at how the dependencies between $a$ and $b$ affect the difficulty in finding $\bar{a}$. In particular, we argue that since the algorithm may not have any knowledge about the parts of the solution that may be reused (i.e., b), the higher the dependency (or lack of modularity) between the 'correct' and 'incorrect' parts of the solution, the more difficult the process of relocating the new global optimum may become.

\section{MODULARITY}

The work carried out in this paper has been inspired, to some extend, by recent studies that investigate the role of modularity in evolving systems. The work by Kashtan et al. [10], [11] studies the effects of dynamic environments on the speed of evolution and the authors find that the presence of dynamics, so long they are modular, has a positive impact on the speed of evolution. In particular, the authors find that "the highest speedup is found under modularly varying goals, in which goals change over time such that each new goal shares some of the subproblems with the previous 
goal." [11, p 13711]. Although the framework employed by the authors is rather simplistic, the results may be very relevant to evolutionary dynamic optimisation. In particular, the authors argue that the speedup is due to local positive gradients in the search space that are generated by new subgoals. More specifically, "local maxima or plateaus in one goal correspond to areas with a positive fitness gradient for the second goal." [11, p 13715]. These results imply that modularity may play an important role in the speed of evolution because it creates a fitness landscape that may be easier to traverse due to a reduced number of local optima and neutral plateaus. It should be noted that modularity is a much researched subject, especially in fields such as evolutionary biology, and here we do not intend to make any statements regarding the definition or impact of modularity in such systems. We restrict ourselves to a simple measure of modularity that simply equates to functional independency (see below).

The problem we consider in this paper is the well-known NK-fitness landscape (NK; [12]) which has been used extensively to model the epistatic effects of genetic regulatory networks. In the $\mathrm{NK}$, each of the $n$ genes (bits) is dependent on itself and $k<n$ other genes. These genes are either chosen to be adjacent to the gene in question or are chosen uniformly at random. Each gene has a fitness table of $2^{k+1}$ entries in the range $[0,1]$ that is indexed using the gene's own state (i.e., $\{0,1\}$ ), as well as the state of the $k$ dependent genes. The fitness values are generate uniformly random and the fitness of a solution is simply the average of all $n$ fitness contributions:

$$
f(x)=\frac{1}{n} \sum_{i=1}^{n} f_{i}\left(x_{i} ; x_{i_{1}}, \ldots, x_{i_{k}}\right)
$$

where $\left\{i_{1}, \ldots, i_{k}\right\} \subset\{1, \ldots, i-1, i+1, \ldots, n\}$. For random neighbourhoods, which are used in this paper, this problem has been shown to be NP-hard for values of $k \geq 2$ (see [1]).

The inter-dependencies between the variables restricts the kind of values each variable may take on (i.e., assignments that improve the fitness of the current search point). For instance, the simple additively decomposable fitness function (see [15]) $f(a, b, c)=f_{0}(a, b)+f_{1}(b, c)+f_{2}(a, c)$ consists of three variables dependent on one another: The values of $a$ and $b$ are dependent on one another, as are the values of $b$ and $c$ and $a$ and $c$. A change in value of any variable may thus trigger a change in any of the dependent variables (if that improves the overall fitness). The lack of such dependencies leads to modular functions where components (i.e., groups of variables) are functionally independent. An example of a modular function would be the function $f(a, b, c, d, e, g)=$ $f_{0}(a, b)+f_{1}(b, c)+f_{2}(a, c)+f_{3}(d, e)+f_{4}(e, g)$ where the variables are split into two functionally independent groups: $\{a, b, c\} \cap\{d, e, g\}=\{\}$. Although there are dependencies within each group, each group may be optimised independently.

\section{DYNAMIC FRAMEWORK}

\section{A. Dynamic Optimisation Problems}

As mentioned in the introduction, the fundamental difference between DOPs and their time-invariant counterparts is time, which affects the mapping from the search space to the domain of real values: $f:\{0,1\}^{n} \times \mathbb{N} \rightarrow \mathbb{R}$. A DOP may be seen as a time series of instances $I$ of the same problem:

$$
I(T) \longrightarrow I(T+1) \longrightarrow I(T+2) \longrightarrow \ldots
$$

where $T$ is the period index $T \cdot \tau \leq t<(T+1) \cdot \tau$ and $\tau$ the update period (i.e., $1 / \tau$ is the frequency of change). Here we only consider a simple class of DOPs and assume there to be no time-linkage (see [3]) between problem instances and that the domain and dimensionality of the search space remains constant over time. In this simplified scenario, the search space $X$ is time-invariant and the dynamics are restricted to the fitness values of all elements $x \in X$. This scenario represents the majority of work in the literature as it corresponds to the models adopted by the three most widely used benchmark generators: Moving Peaks [5], DF1 [13] and $X O R$ [20]. An algorithm used to solve such problems observes these changes (i.e., the transition from one instance to another) as alterations to the fitness landscape it traverses. The fitness landscape is specified by the tuple $\mathcal{L}=(X, d, f)$ where $X$ is the search space, $d$ a neighbourhood operator for the elements in $X$ and $f$ is the objective function. The effect of the underlying dynamics, including the potential displacement of the global optimum, thus differ depending on the algorithm (representation, variation operators) used.

In this paper we consider the class of pseudo-Boolean functions $f:\{0,1\}^{n} \rightarrow \mathbb{R}$ where the distance between any two points in this $n$-dimensional search space is given by the Hamming distance $d(x, y)=\sum_{i=1}^{n}\left|x_{i}-y_{i}\right|$. We denote the number of 1-bits in $x$ as $|x|_{1}$. The use of pseudoBoolean functions allows us to make use of a well established dynamic framework which models any pseudo-boolean stationary problem as a dynamic one. The XoR benchmark [19], [20] imposes dynamics on any stationary function by means of a bit-wise exclusive-or operation that is applied to each search point prior to each function evaluation. This operation rotates the search point, simulating the displacement that may occur from fitness re-scalings (e.g., a shifting peak). The dynamic equivalent of any stationary function is simply $f\left(x(t) \oplus m_{d}(T)\right)$ where $\oplus$ is the xor operator. The vector $m_{d}(T) \in\{0,1\}^{n}$, initially $0^{n}$, is a binary mask that is continuously generated as $m_{d}(T):=m_{d}(T-1) \oplus p(T)$ where $p(T) \in\{0,1\}^{n}$ is a randomly created template that contains exactly $\lfloor\rho n\rfloor$ ones. In other words, for each period $T$, the mask $m_{d}$ is altered using a randomly created template $p$ that encapsulates the magnitude of change $\rho \in(0,1]$ (i.e., $\rho n$ is the actual number of bits inverted). In this work, we denote the magnitude of change as an integer $d$ that corresponds to the number of bits affected by XoR: $0<d \leq n$. 


\section{B. Dynamic Modular Framework}

The modular framework we introduce here makes use of similar concepts employed by XoR. The goal was to create a framework that may be implemented and replicated easily to study the impact of modularity on the performance of an algorithm used to solve a particular DOP. The framework has clear limitations, which we will discuss shortly, but introduces dependencies between variables without altering the complexity of the underlying function. Subsequently, the impact of modularity (or lack thereof) is isolated and solely accounts for any observable differences in the algorithm's behaviour.

Given some pseudo-Boolean function, a bi-modular variant is created as follows: The two modules, $a$ and $b$, are defined as independent functions $f_{a}(x)$ and $f_{b}(x)$ and the overall function, $F(x)$ is simply the average output of both functions:

$$
F(x)=\frac{f_{a}(a)+f_{b}(b)}{2}
$$

A solution to $F$ consists of a prefix and a suffix, $x=a b$, such that $|a|+|b|=|x|=n$ and for now we assume that $|a|=$ $|b|$. The degree of interaction between these independent functions is introduced using a mask $m_{m} \in\{0,1\}^{|a|}$ and the logical and operand:

$$
F(x)=\frac{\left.f_{a}(a)+f_{b}\left(\left(a \wedge m_{m}\right) \oplus b\right)\right)}{2}
$$

where $\left|m_{m}\right|_{1}$ determines the degree of overlap between the modules: A value of $m_{m i}=0$ implies a lack of dependency between $a_{i}$ and $b_{i}$ such that any changes to $a_{i}$ do not affect the status of $b_{i}$. On the other hand, a value of $m_{m i}=1$ implies that the bit $b_{i}$ is affected every time $a_{i}$ changes its value (see example below).

The actual dynamics are modelled using the same exclusive-or operation introduced earlier such that the function is now fully defined as:

$$
F(x, t)=\frac{\left.f_{\alpha}\left(a \oplus m_{d}(t)\right)+f_{\beta}\left(\left(a \wedge m_{m}\right) \oplus b\right)\right)}{2}
$$

The impact of these operations may be illustrated as follows: We assume the global optima $x_{f_{a}}^{\star}=1100$ and $x_{f_{b}}^{\star}=$ 0011, a degree of modularity of $m=2$ and a magnitude of change of $d=2$. The mask $m_{m}$ may be generated randomly as long as $\left|m_{m}\right|=m$ and here we assume $m_{m}=0101$. The mask $m_{d}$ equals 1010 in this example. Given the algorithm has located both global optima, the solution for $f_{a}$ found by the algorithm is $a=1100 \oplus 1010=0110$. The mask $m_{m}$ is therefore $m_{m}=0110 \wedge 0101=0100$ and subsequently, $b=0011 \oplus 0100=0111$. If a change takes place, the mask $m_{d}$ is modified randomly such that $d\left(m_{d}(T), m_{d}(T+1)\right)=$ $d$. Let us assume the new mask is $m_{d}(T+1)=0011$. This means that the bits $a_{1}$ and $a_{4}$ need to be inverted to relocate the global optimum (i.e., $a=1111$ ). The change in value of $a_{1}$ will not affect $b_{1}$ as $m_{m 1}=0$. However, once the algorithm inverts $a_{4}$, the value of $b_{4}$ is affected such that the new points found by the algorithm are $a=1111$ and $b=0110$. Therefore, although the dynamics only affect the prefix of the solution, the dependency between the modules required a change in $b$ by 1 bit.

So far, we have only considered the case where $|a|=$ $|b|$. It is possible to generalise the framework to arbitrary module sizes although this requires a mapping to adjust for any difference in size. If, for instance, $|a|=\frac{1}{2}|b|$, the mask $m_{m}$ needs to be doubled in size to yield $\hat{m}_{m}$ where $\left|\hat{m}_{m}\right|=$ $|b|$. The new mask $\hat{m}_{m}$ is based on $m_{m}$ and the way it is generated allows for different types (severities) of modularity to be modelled. In the simplest case, and assuming that $|b|>$ $|a|, \hat{m}_{m}=m_{m} 0^{|a|}$ (i.e., the mask simply gets extended by adding 0 s to the end). The value 0 does not cause any impact on $b$ and hence the impact of $a$ is restricted to the first $|a|$ bits of $b$; this is the approach adopted in this paper. Another approach may be to simply multiply the value of each bit in $a$ by $|b| /|a|$, consecutively copying the bits from $a$ to $b$. For instance, if $a=11000$ (i.e., $|a|=5$ ) and $|b|=10$, we could produce a mask $\hat{m}_{m}=1111000000$. Here, every bit position $i$ in $a$ occupies bit positions $j$ and $j+1$ in $b$ where $j=2 i$. This would increase the influence of $a$ over $b$.

This approach to modularity is clearly very simple and has some limitations. The probably biggest problem is the direction of the dependencies as only elements in a may affect elements in $b$ but not vice versa. It would be possible to extend the model to have two masks, $m_{m_{a}}$ and $m_{m_{b}}$, one for $a$ and one for $b$. However, any dependencies that would form a cycle would prevent the algorithm from making progress: If bit $a_{i}$ affects bit $b_{j}$, which in turn affects bit $a_{i}$, any changes to $a_{i}$ cause $b_{j}$ to change which causes $a_{i}$ to change and so on. On the other hand, it is possible to account for more than two modules and to define more complex dependencies between those modules as long as such cyclical dependencies are avoided. Nevertheless, despite these limitations, this approach, similar to XoR, allows one to precisely model very specific effects. For instance, linkage between two variables does not necessarily imply that if one value changes, so does the other. In the proposed framework, this is always the case and hence allows one to precisely specify the degree of dependency of one variable on another (as an upper bound).

\section{Algorithm}

The algorithm we consider is the well-known $(1+1)$ EA as shown in Figure 1. The (1+1) EA only manipulates a single solution at a time (i.e., no population) and uses a bit-wise mutation operator to generate new search points. EAs are usually considered promising candidates for DOPs because of their use of populations. However, we have chosen the $(1+1)$ EA as it allows us to precisely define the initial search point which is not possible with populations that are not fully converged $^{2}$. The $(1+1)$ EA as shown in Figure 1 as been adapted by Droste to be used on DOPs [6]. The difference between the traditional algorithm and its counterpart for the dynamic domain is the inclusion of time. In particular, in

\footnotetext{
${ }^{2}$ It is possible to define distributions of points around a single initial search point which is something we intend to look at in the near future.
} 


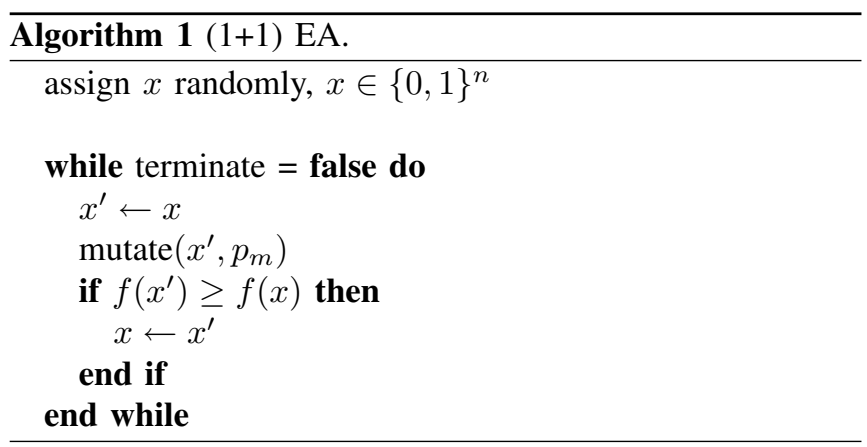

the dynamic case, the algorithm evaluates both search points (i.e., parent and offspring) during each time step (iteration) to ensure no outdated fitness values are used.

\section{EXPERIMENTAL SETUP}

The underlying problem used to create the modular function is the $\mathrm{NK}$ with various values for $k$. The dimension of the problem is limited to $n=15$ and although such instances are very small in size, the need to exhaustively sample the search space (in order find the global optimum) negates the possibility of larger instances. We assume the algorithm has found the global optimum, followed by a change induced using XoR. The magnitudes of change considered are $d \in$ $\{1,2,3,4,5\}$. The bits that are incorrect following a change will be labelled $a$ (and the correct bits will be labelled $b$; see section II). We then look at different levels of dependency between $a$ and $b$ to determine the time it takes the algorithm to successfully find $\bar{a}$. We treat $a$ as a module functionally independent of $b$ and then create dependencies using $m_{m}$ with values of $m \in\{0,1, \ldots,|a|\}$. The mask $\hat{m}$ is created by simply adding $|b|-|a|$ 0-bits at the end (see section IV-B). The values for $k$ are chosen to be identical for $a$ and $b$ such that $k_{a}=k_{b} \in\{0,1,2\}\left|k_{a}=k_{b}<\right| a \mid$. We set a limit on the number of function evaluations allowed to $2^{n}=32768$ and record the number of times the global optimum has been found within that bound. In the second set of experiments, we look at the algorithm's performance over time. For this, we plot the fitness values over time as the function undergoes 5 changes with 200 function evaluations in between changes. The values are as follows: $k_{1}=k_{b}=1, d \in\{2,3,4,5\}$ and $m=1,2, \ldots, d$. In all cases, we average over 50 trials.

\section{RESULTS}

The results for the first set of experiments are shown in Table I. The Table shows the success rates of the $(1+1)$ EA on the bi-modular $n=15 \mathrm{NK}$, starting either from a specific distance to the global optimum or from a randomly chosen point. If $k$ is 0 , the success rate is always 1 as the underlying problem is very simple ("Fujiyama landscape"; see [12]). As $k$ increases, the success rates drop for any distance d. Likewise, the success rate drops, as expected, as the initial distance to the new global optimum increases. More interestingly, however, is the relationship between the degree of modularity and the success rate. Here it is evident from the data that the success rate is not only reduced by a lack of modularity but that this decrease is correlated to the value of $k$. In other words, decreasing the degree of modularity decreases the success rate, at a rate that is faster the higher the value of $k$. Looking at the case of $d=5$, for instance, we see that for $k=0$, the success rate remains the same across all values for $m$. For the other two values of $k$, on the other hand, the following trends are evident:

$\begin{array}{ccccccc} & 0 & 1 & 2 & 3 & 4 & 5 \\ k=1 & 0.98 & 0.93 & 0.90 & 0.85 & 0.83 & 0.79 \\ k=2 & 0.86 & 0.78 & 0.72 & 0.63 & 0.50 & 0.41\end{array}$

The reasons for this are as follows: Whenever an element in $a$ changes that is linked to an element in $b$, the corresponding element in $b$ is required to change its value as well. Elements in $b$ are dependent to one another as controlled by $k$ and whenever a change in $a$ forces a change in $b$, other bits in $b$ may be indirectly affected. Furthermore, if these bits also happen to be dependent upon elements in $a$, it could be that they change their value multiple times depending on the order and speed at which the elements in $a$ are inverted. A higher value of $k$ not only slows down the inversion of $a$ but also implies that any change in $b$ resulting from changes in $a$ may propagate to other bits in $b$.

Table I also shows the success rate for the $(1+1)$ EA starting from a randomly chosen search point. There are two interesting trends to observe: In some cases, the restart approach has a lower success rate, implying that reusing the old global optimum as an initial search point is beneficial. However, as the magnitude of change increases, the success rates of the random restart approach becomes superior, even if the distance remains relatively minor. This superiority is also related to the degree of overlap between the modules as the differences increase in proportion to increasing values of $m$. Subsequently, even for a distance of $d=4$ a random restart may perform superior if the dependency between the correct and incorrect bits is large.

The performance of the $(1+1)$ EA over time is shown in Figure 1 for different values of $m$ and $d$. Such best of generation graphs are commonly used to visualise the performance of an EA on a DOP. It is evident that the degree of overlap between the problem's modules affects the rate at which the algorithm is able to re-locate the global optimum. For all distances considered, a higher degree of overlap always results in a reduced performance: Although the initial reduction in fitness is identical across all cases, the speed at which fitness is regained is higher in those cases where $a$ may be optimised independently of $b$. Table II summarises the performance values for each run (which is simply the average of all f-values over time). In general, the numerical differences are rather small but this is largely due to the nature of NK. As expected, the performance degrades in proportion to the magnitude of change and the degree of overlap. It is interesting to note that similar values are obtained for different settings. For instance, the performance for $d=3$ and $m=3$ is 0.9486, similar to the case $d=5$ and $m=1(0.9484)$. This similarity is due to the overall (effective) distance that the algorithm is faced with: 
TABLE I

SUCCESS RATES FOR $d \in\{1,2,3,4,5\}, m=1, \ldots, d$ AND

$k=0,1, \ldots, d-1$. BOLD VALUES INDICATE THAT THE RANDOM RESTART APPROACH IS SUPERIOR.

\begin{tabular}{|c|c|c|c|c|c|}
\hline$d$ & $m$ & $k$ & non-random & random & difference \\
\hline 1 & 0 & 0 & 1.00 & 1.00 & \\
\hline 1 & 1 & 0 & 1.00 & 1.00 & \\
\hline 2 & 0 & 0 & 1.00 & 1.00 & \\
\hline 2 & 0 & 1 & 1.00 & 0.95 & 0.04 \\
\hline 2 & 1 & 0 & 1.00 & 1.00 & \\
\hline 2 & 1 & 1 & 0.99 & 0.95 & 0.04 \\
\hline 2 & 2 & 0 & 1.00 & 1.00 & \\
\hline 2 & 2 & 1 & 0.97 & 0.94 & 0.03 \\
\hline 3 & 0 & 0 & 1.00 & 1.00 & \\
\hline 3 & 0 & 1 & 1.00 & 0.97 & 0.03 \\
\hline 3 & 0 & 2 & 0.98 & 0.79 & 0.18 \\
\hline 3 & 1 & 0 & 1.00 & 1.00 & \\
\hline 3 & 1 & 1 & 0.99 & 0.97 & 0.02 \\
\hline 3 & 1 & 2 & 0.91 & 0.78 & 0.13 \\
\hline 3 & 2 & 0 & 1.00 & 1.00 & \\
\hline 3 & 2 & 1 & 0.99 & 0.96 & 0.02 \\
\hline 3 & 2 & 2 & 0.85 & 0.76 & 0.08 \\
\hline 3 & 3 & 0 & 1.00 & 1.00 & \\
\hline 3 & 3 & 1 & 0.89 & 0.93 & 0.04 \\
\hline 3 & 3 & 2 & 0.75 & 0.74 & 0.01 \\
\hline 4 & 0 & 0 & 1.00 & 1.00 & \\
\hline 4 & 0 & 1 & 0.98 & 0.95 & 0.03 \\
\hline 4 & 0 & 2 & 0.94 & 0.80 & 0.14 \\
\hline 4 & 1 & 0 & 1.00 & 1.00 & \\
\hline 4 & 1 & 1 & 0.94 & 0.93 & 0.01 \\
\hline 4 & 1 & 2 & 0.86 & 0.77 & 0.09 \\
\hline 4 & 2 & 0 & 1.00 & 1.00 & \\
\hline 4 & 2 & 1 & 0.90 & 0.91 & 0.01 \\
\hline 4 & 2 & 2 & 0.74 & 0.72 & 0.02 \\
\hline 4 & 3 & 0 & 1.00 & 1.00 & \\
\hline 4 & 3 & 1 & 0.85 & 0.88 & 0.03 \\
\hline 4 & 3 & 2 & 0.67 & 0.71 & 0.03 \\
\hline 4 & 4 & 0 & 1.00 & 1.00 & \\
\hline 4 & 4 & 1 & 0.75 & 0.86 & 0.11 \\
\hline 4 & 4 & 2 & 0.62 & 0.68 & 0.06 \\
\hline 5 & 0 & 0 & 1.00 & 1.00 & \\
\hline 5 & 0 & 1 & 0.98 & 0.98 & \\
\hline 5 & 0 & 2 & 0.86 & 0.75 & 0.10 \\
\hline 5 & 1 & 0 & 1.00 & 1.00 & \\
\hline 5 & 1 & 1 & 0.93 & 0.96 & 0.03 \\
\hline 5 & 1 & 2 & 0.78 & 0.74 & 0.04 \\
\hline 5 & 2 & 0 & 1.00 & 1.00 & \\
\hline 5 & 2 & 1 & 0.90 & 0.95 & 0.05 \\
\hline 5 & 2 & 2 & 0.72 & 0.72 & \\
\hline 5 & 3 & 0 & 1.00 & 1.00 & \\
\hline 5 & 3 & 1 & 0.85 & 0.92 & 0.07 \\
\hline 5 & 3 & 2 & 0.63 & 0.70 & 0.07 \\
\hline 5 & 4 & 0 & 1.00 & 1.00 & \\
\hline 5 & 4 & 1 & 0.83 & 0.91 & 0.08 \\
\hline 5 & 4 & 2 & 0.50 & 0.66 & 0.17 \\
\hline 5 & 5 & 0 & 1.00 & 1.00 & \\
\hline 5 & 5 & 1 & 0.79 & 0.90 & 0.11 \\
\hline 5 & 5 & 2 & 0.41 & 0.63 & 0.22 \\
\hline
\end{tabular}

$3+3=5+1$. In fact, it may be observed how across the diagonal (where the values $d+m$ are identical), all performances are very similar to one another.

Despite these similarities, it should be noted that the framework used in this paper is not the same as simply extending the magnitude of change (using only XoR) for the same reasons as explained above (i.e., the path taken to the global optimum in the modular case may ultimately be longer than the path taken otherwise). Table III shows the
TABLE II

OFFLINE PERFORMANCE FOR $d \in\{2,3,4,5\}, m=1, \ldots, d$ AND $k=1$.

\begin{tabular}{c|cccc}
\hline $\mathrm{m}$ & 2 & 3 & 4 & 5 \\
\hline \hline 0 & 0.9656 & 0.9599 & 0.9551 & 0.9520 \\
1 & 0.9627 & 0.9567 & 0.9514 & 0.9484 \\
2 & 0.9593 & 0.9528 & 0.9478 & 0.9450 \\
3 & & 0.9486 & 0.9436 & 0.9408 \\
4 & & & 0.9401 & 0.9375 \\
5 & & & & 0.9340 \\
\hline
\end{tabular}

TABLE III

OFFLINE PERFORMANCE FOR $d \in\{3,4,5\}, m=1, \ldots, d$ AND $k=2$.

\begin{tabular}{c|ccc}
\hline $\mathrm{m}$ & 3 & 4 & 5 \\
\hline \hline 0 & 0.9372 & 0.9353 & 0.9361 \\
1 & 0.9329 & 0.9310 & 0.9332 \\
2 & 0.9287 & 0.9278 & 0.9305 \\
3 & 0.9242 & 0.9239 & 0.9267 \\
4 & & 0.9195 & 0.9239 \\
5 & & & 0.9210 \\
\hline
\end{tabular}

same data as Table II but for $k=2$ and here the values across the diagonal do vary, indicating that the distance $d+m$ is, by itself, not sufficient to account for the differences in the algorithm's behaviour observed.

In [14], we distinguished between the actual and observed magnitude of change. The data here shows evidence for the effective magnitude of change. Similar to the concept of a point's effective distance ([2]; see section II), the effective magnitude of change correlates the time it may take to relocate the global optimum to the actual (genotype) distance between successive global optima. The three concepts relate as follows: The dynamics structurally alter some aspects of the problem (actual dynamics), causing the fitness landscape to change depending on the representation and variation operators used by the algorithm (observed dynamics). Finally, the time it takes the algorithm to relocate the global optimum corresponds to the effective distance.

\section{CONCLUSIONS}

Evolutionary dynamic optimisation is a rapidly growing field of research that is concerned with the application of evolutionary algorithms (EAs) to dynamic optimisation problems (DOPs). One of the fundamental goals of this field of research is to reduce the computational cost associated with relocating the function's global optimum once it has moved due to the underlying dynamics of the problem. The major strategy employed by the majority of techniques is the transfer of knowledge from one problem instance in time to the next. For the majority of approaches, this transfer of knowledge is restricted to the initial search point (or search points in the case of populations) that is supplied to the algorithm. This search point is expected to be closer to the global optimum than a randomly generated initial search point, potentially granting the algorithm some advantage. However, relatively little is known about the type of scenarios in which this approach is effective and in this paper, we have 


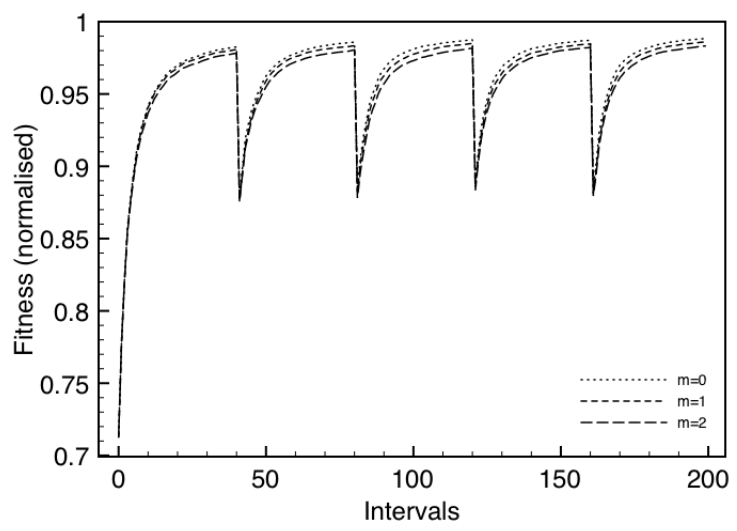

(a)

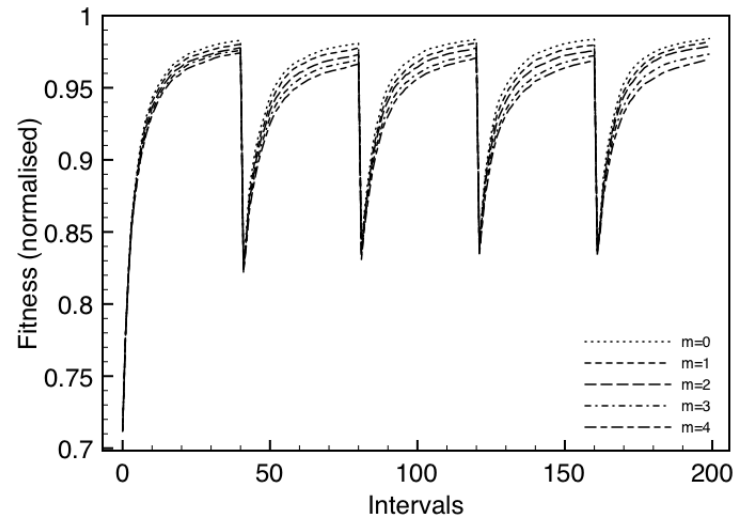

(c)

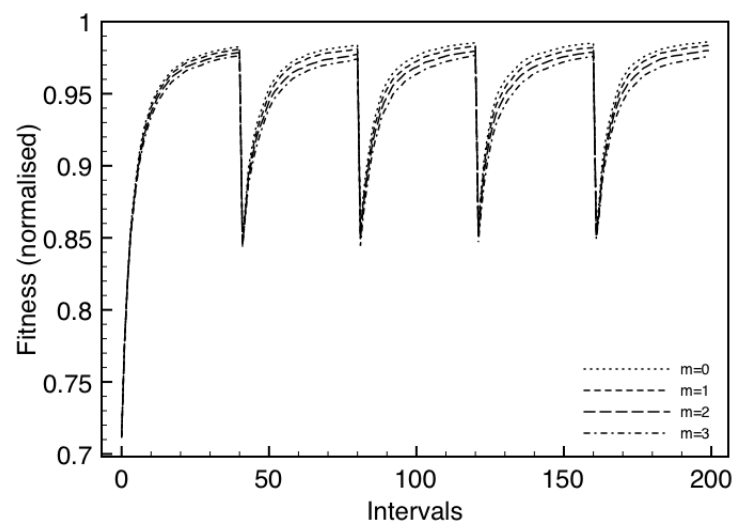

(b)

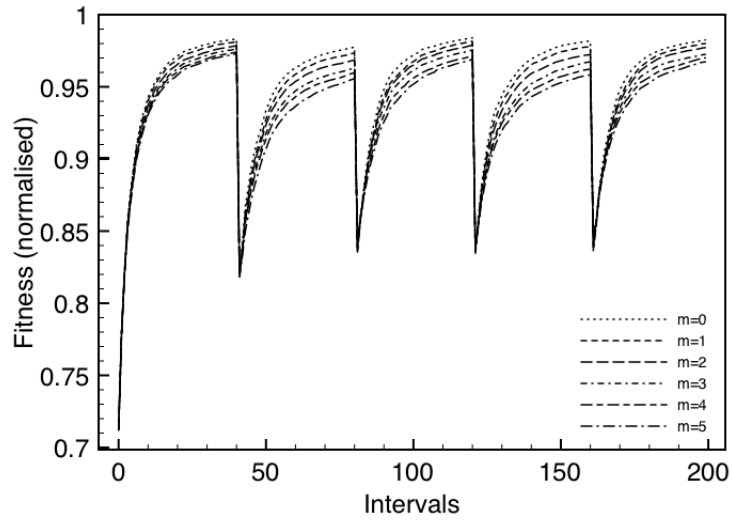

(d)

Fig. 1. Performances for $k=1$, (a) $d=2$, (b) $d=3$, (c) $d=4$, (d) $d=5$ and $m=0,1, \ldots, d$.

looked at the impact of modularity on the time it takes the algorithm to relocate the global optimum.

The results have shown how the effective distance between successive global optima may differ from the observed distance depending on the structural properties of the problem. In particular, of one views the moving global optimum as a transformation from a string $x(T)=a b$ to a string $x(T+$ $1)=\bar{a} b$, where $\bar{a}$ is the inverse of $a$, the dependency between $a$ and $b$ has a significant impact on the time it takes the algorithm to invert all incorrect bits (i.e., a). We highlighted this relationship by suggesting a simple framework that may be used to turn any pseudo-Boolean function into a modular one and using the well-known NK fitness landscape as a base problem. Within this framework, inverting any of the incorrect bits (i.e., getting closer to the global optimum), may require a subsequent alteration of some of the previously correct bits. This dependency is propagated further by the dependencies between bits within each module (i.e., the value of $k$ in the case of $\mathrm{NK}$ ).

There are numerous extensions to this work that we intend to carry out in the near future. In particular, we believe it would be very interesting to extend this work to other problems such as the dynamic travelling salesman problem, using real-world data where available, to determine whether modularity is found in such cases. It might then be promising to investigate linkage learning techniques that may be used to identify components of the underlying problem that may be reused in their entirety whenever a change occurs. This could significantly speed up the rate at which the algorithm relocates the global optimum. Also, modularity might play a crucial role in the displacement of the global optimum in the first place. In [14], we highlighted the relationship between the underlying magnitude of change (i.e., changes to the problem's parameters) and the movement of the global optimum. The results showed that even small changes may have an arbitrarily large impact on the position of the global optimum depending on the structural properties of the instance's fitness landscape (fitness distance correlation in this case). Modularity may greatly influence this displacement as changes to functionally isolated parts of the problem would have a limited overall effect on the structural properties of the fitness landscape. Finally, it would also be interesting to strengthen the links to studies of biological systems where modularity seems to play a vital role in the speed and ability of a species to adapt.

\section{ACKNOWLEDGMENTS}

This work was supported by EPSRC grant no. EP/E058884/1 entitled "Evolutionary Algorithms for Dynamic Optimisation Problems: Design, Analysis and Applications". 


\section{REFERENCES}

[1] L. Altenberg. NK fitness landscapes. In T. Bäck, D. B. Fogel, and Z. Michalewicz, editors, The Handbook of Evolutionary Computation, pages B2.7:2-B2.7:10. Oxford University Press, 1997.

[2] Y. Borenstein and R. Poli. Information landscapes. In GECCO 05: Proceedings of the 2005 conference on Genetic and evolutionary computation, pages 1515-1522, New York, NY, USA, 2005. ACM.

[3] P. A. N. Bosman. Learning, anticipation and time-deception in evolutionary online dynamic optimization. In Proceedings of the 2005 workshops on Genetic and Evolutionary Computation, pages 39-47, 2005.

[4] J. Branke. Evolutionary approaches to dynamic environments - updated survey. In GECCO Workshop on Evolutionary Algorithms for Dynamic Optimization Problems, pages 27-30, 2001.

[5] J. Branke. Evolutionary Optimization in Dynamic Environments. Kluwer, 2002.

[6] S. Droste. Analysis of the $(1+1)$ ea for a dynamically changing objective function. Technical report, Universitt Dortmund, 2001.

[7] S. Droste, T. Jansen, and I. Wegener. Optimization with randomized search heuristics - the (a)nfl theorem, realistic scenarios, and difficult functions. THEORETICAL COMPUTER SCIENCE, 287, 2002.

[8] Y. Jin and J. Branke. Evolutionary optimization in uncertain environment - a survey. IEEE Transactions on Evolutionary Computation, 9(3):303-317, 2005.

[9] T. Jones and S. Forrest. Fitness distance correlation as a measure of problem difficulty for genetic algorithms. In Proceedings of the Sixth International Conference on Genetic Algorithms, pages 184-192, 1995.

[10] N. Kashtan and U. Alon. From the cover: Spontaneous evolution of modularity and network motifs. Proc Natl Acad Sci USA, 102(39):13773-13779, 2005.

[11] N. Kashtan, E. Noor, and U. Alon. Varying environments can speed up evolution. PNAS, 104(34):13711-13716, 2007.

[12] S. A. Kauffman. The Origins of Order. Oxford University Press, 1993.

[13] R. W. Morrison. Designing Evolutionary Algorithms for Dynamic Environments. Springer, Berlin, 2004.

[14] P. Rohlfshagen and X. Yao. Attributes of dynamic combinatorial optimisation. In Lecture Notes in Computer Science, volume 5361/2008, pages 442-451. Springer, 2008.

[15] R. Thompson and A. Wright. Additively decomposable fitness functions. Technical report, University of Montana, Computer Science Department, 1996.

[16] I. Wegener. Methods for the analysis of evolutionary algorithms on pseudo-boolean functions. Evolutionary optimization, pages 349-369, 2002.

[17] K. Weicker. Evolutionary Algorithms and Dynamic Optimization Problems. Der Andere Verlag, 2003.

[18] D. H. Wolpert and W. G. MacReady. No free lunch theorems for optimization. IEEE Transactions on Evolutionary Computation, 1(1):67-82, April 1997.

[19] S. Yang. Non-stationary problem optimization using the primal-dual genetic algorithms. In R. Sarker, R. Reynolds, H. Abbass, K.-C. Tan, R. McKay, D. Essam, and T. Gedeon, editors, Proceedings of the 2003 IEEE Congress on Evolutionary Computation, volume 3, pages 22462253, 2003.

[20] S. Yang. Memory-based immigrants for genetic algorithms in dynamic environments. In Proceedings of the 2005 Genetic and Evolutionary Computation Conference, volume 2, pages 1115-1122. ACM Press, 2005. 